Meta

Journal des traducteurs

Translators' Journal

\title{
Au dossier de « nursing » (suite)
}

\section{Jacques Boulay}

Volume 13, numéro 1, mars 1968

URI : https://id.erudit.org/iderudit/002123ar

DOI : https://doi.org/10.7202/002123ar

Aller au sommaire du numéro

Éditeur(s)

Les Presses de l'Université de Montréal

ISSN

0026-0452 (imprimé)

1492-1421 (numérique)

Découvrir la revue

Citer cet article

Boulay, J. (1968). Au dossier de "nursing " (suite). Meta, 13(1), 24-25.

https://doi.org/10.7202/002123ar

Ce document est protégé par la loi sur le droit d'auteur. L’utilisation des services d'Érudit (y compris la reproduction) est assujettie à sa politique d'utilisation que vous pouvez consulter en ligne.

https://apropos.erudit.org/fr/usagers/politique-dutilisation/
Cet article est diffusé et préservé par Érudit.

Érudit est un consortium interuniversitaire sans but lucratif composé de l'Université de Montréal, l'Université Laval et l'Université du Québec à Montréal. Il a pour mission la promotion et la valorisation de la recherche. https://www.erudit.org/fr/ 


\section{AU DOSSIER DE “NURSING" (SUITE)}

Le docteur Jacques Boulay, secrétaire général du Comité d'étude des termes de médecine, nous adresse la lettre suivante:

Dans le numéro de septembre 1967 de la revue Meta, Mademoiselle Adrienne Audette [... ] tente de justifier, après bien d'autres, l'emploi du mot «nursing». Puisque le Comité d'étude des termes de médecine, dont j’ai l'honneur d'être le secrétaire général, a condamné sans équivoque cet anglicisme, d'accord en cela avec le Comité d'étude des termes médicaux français et la Commission consultative de l'Office de la langue française du Québec, je vous saurais gré de m'accorder quelques lignes pour relever les sophismes de votre correspondante et préciser la position de notre Comité sur cette question.

Pour Mademoiselle Audette, le mot «nursing» n'a pas d'équivalent exact en français, ce qui justifierait son adoption dans notre langue. Si Mademoiselle Audette est traductrice, elle doit savoir qu'il y a, dans toute langue de départ, un très grand nombre de mots et d'expressions qui n'ont pas d'équivalent exact dans la langue d'arrivée, et ceci parce que chaque langue découpe la réalité à sa manière. Si l'on exigeait d'avoir des équivalents exacts pour chaque terme ou groupe de termes à traduire, la traduction serait impossible. Or elle est possible, puisqu'elle existe.

Ceci étant dit, est-il bien vrai que l'expression soins infirmiers ne rend pas tout le contenu du mot «nursing»? Rien n'est moins sûr, quoi qu'en pensent les médecins français que cite Mademoiselle Audette. Les docteurs J.-J. Rondepierre, Pierre Joannon et M. Audier, sur l'autorité desquels Mademoiselle Audette s'appuie, sont d'illustres inconnus dans le domaine du langage médical, et je présume qu'il ne suffit pas d'être français pour pouvoir se prononcer avec autorité sur une question de terminologie. Sur ce point, on peut résumer la pensée de votre collaboratrice de la façon suivante: le «nursing», c'est l'ensemble de tous les actes que peut accomplir une infirmière pour le bien-être de son malade. Or pourquoi ces actes ne pourraient-ils pas être désignés du nom générique de soins? Le mot soins ne désigne-t-il pas les actes par lesquels on soigne quelqu'un, quelque chose (Robert, article soins, $4^{\circ}$ ) et non seulement les actions par lesquelles on conserve ou on rétablit la santé? Pour une infirmière, déplacer un oreiller sous la tête d'un malade, donner un lavement, faire une piqûre, prendre la température et la noter, dire une parole réconfortante, accorder un sourire, constituent des soins. Ne dit-on pas d'une infirmière qu'elle soigne bien ses malades ou qu'elle en prend bien soin?

15. "Etude de quelques termes mis en circulation par la télévision », META, vol. XI, no 2 , juin 1966, p. 64. 
D'autre part, je ne vois pas pourquoi les observations que l'infirmière fait sur ses malades, et auxquelles, paraît-il, Florence Nightingale donnait beaucoup d'importance, ne pourraient pas relever des soins infirmiers, car il esi bien évident que tout geste destiné à donner plus de bien-être au malade a son point de départ dans une observation. Et les infirmières de langue française, qui soignaient des malades avant que le "nursing 》 ne vînt rehausser leur prestige, ne procédaient pas différemment.

Je pense que la préférence que donne Mademoiselle Audette à «nursing》 est d'origine para-linguistique. Les infirmières, celles du Québec en particulier, veulent accroître leur prestige - ce qui n'est que louable; pour ce faire, elles empruntent un terme qui a cours au sein de la collectivité à laquelle elles voudraient inconsciemment s'identifier [... ]. Elles ne soignent plus les malades, elles font du «nursing »; bientôt, elles ne s'appelleront plus infirmières, mais spécialistes en nursing ou «nursingistes», comme «diététistes». Et cette maladie de la langue qu'est l'emprunt systématique n'existe pas qu'au Québec, elle se répand aussi en France. Ce sont les mêmes mécanismes psycholinguistiques qui poussent les Français à dire "charter » plutôt qu'avion nolisé ou affrété et les infirmières de chez nous à dire «nursing 》 plutôt que soins infirmiers.

Vous conviendrez avec moi qu'il est assez désolant de constater que certains spécialistes du langage, malgré leur connaissance des phénomènes qui sous-tendent la langue, encouragent cet asservissement de l'esprit que manifeste l'envahissement de notre idiome par des vocables aussi inutiles qu'inesthétiques, au premier rang desquels figure le mot «nursing». Heureusement, il y en a d'autres qui sont plus conscients de leurs responsabilités et dont la compétence est d'ailleurs notoire - je pense aux membres de la Commission consultative de l'Office de la langue française, en particulier à $M$. Jean Darbelnet. Forts de l'autorité que nous donne l'approbation de ces linguistes, nous continuerons donc à nous battre pour la disparition de «nursing».

P.S. Mademoiselle Audette pourra lire les numéros de la dernière année de la reviue l'Infirmière canadienne pour constater à quel point le vocabulaire des soins infirmiers que nous avons proposé est capable de décrire la réalité nord-américaine d'une façon adéquate. Le mot "nursing y a été supprimé systématiquement, et la Revue n'en a que plus belle allure.

JACQUES BOULAY 